A simple method for the simulation of steady-state diffusion through membranes: pressure-tuned, boundary-driven molecular dynamics

Zoltán Ható ${ }^{1}$, Ákos Kaviczki ${ }^{2}$ and Tamás Kristóf ${ }^{3} *$

Institute of Chemistry, Department of Physical Chemistry, University of Pannonia, Veszprém, Hungary

P.O. Box 158, H-8201 Veszprém, Hungary

${ }^{1}$ hato@almos.uni-pannon.hu

2 akaviczki@gmail.com

${ }^{3}$ kristoft@almos.uni-pannon.hu

* corresponding author 


\title{
A simple method for the simulation of steady-state diffusion: pressure-tuned, boundary-driven molecular dynamics
}

\begin{abstract}
We present a novel molecular dynamics based simulation technique for investigating gas transport through membranes. In our simulations the main control parameters are the partial pressure for the components on the input side of the membrane and the total pressure on the output side. The essential point of our scheme is that this pressure control should be realised by adjusting the particle numbers in the input and output side control cells indirectly. Although this perturbation is applied sufficiently far from the membrane, the bulk-phase properties are well controlled in a simulation cell of common size. Numerical results are given for silicalite-1 membrane with permeating $\mathrm{CH}_{4}, \mathrm{CO}_{2}, \mathrm{H}_{2}$ and $\mathrm{N}_{2}$ gases as well as with binary mixtures of $\mathrm{CO}_{2}$ with the other three components. To describe interactions between particles, we used the simple shifted and cut Lennard-Jones potential with parameters available in the literature. It is expected that the proposed technique can be applied to several other types of membranes and transported fluids in order to support the development of a deeper understanding of separation processes.
\end{abstract}

Keywords: molecular dynamics, steady state, silicalite membrane, gas permeation

\section{Introduction}

Understanding and describing adsorption and diffusion phenomena are critical to design efficient separation methods [1], and molecular simulation has become a very powerful tool to investigate these phenomena [2-8]. The findings from atomistic simulations can explain or in some cases substitute the experimentally obtained results. In this work we will focus on simulating steadystate transport of molecules through membrane. For this we have to provide a method to handle the dynamics of the system and a method to ensure and maintain the steady-state driving force at microscopic level. The dynamics of the system in question can be investigated microscopically by means of molecular dynamics (MD), dynamic Monte Carlo (DMC) [5], Langevin dynamics, 
and other direct or indirect (e.g. NP+LEMC [9]) simulation methods. Today the above procedures are generally coupled to other techniques, e.g. dual control volume (DCV) or local equilibrium Monte Carlo (LEMC) techniques to ensure the constant particle-motive force [8].

The diffusion phenomena through membranes are widely studied in different fields of the simulation community. There are many techniques, with many limits of applicability and many advantages and disadvantages. The gradient relaxation MD proposed by Maginn [10] and a similar approach by Salih [11] is based on the linear response theory to determine diffusion coefficients. The weaknesses are that these simulations are limited by the transient nature of these simulations and that the results have to be fitted to a diffusion equation. The self-adjusting plates technique [12] is suited for simulating transport across membranes, where walls of control cells are moving with constant force applied to them resulting in squeezing the particles through the membrane. This method also suffers from transient effects; the particle numbers are continuously decreasing in the feed side reservoir and the feed side composition can also change due to the selectivity of the membrane (in the case of a mixture, e.g. the fluxes of the components are different). External field MD (also called earlier as colour field MD) can be a good alternative to overcome the depletion phenomena and the transient behaviour [13]. Applying additional force or additional velocities to particles and combining this with periodic boundary conditions in the direction of the transport are the main ideas of this approach. The size and position of the region where the external field is applied in the simulation box may vary from a full control cell to a thin wall-like region [14] far from the transport region. Its novel boundary-driven version [15] is interesting and can be effective in some cases. In our view, however, using an external field is a too direct approach to control the velocities of particles, and combining with periodic conditions in transport direction can result in incorrect density profiles. Also, the external field can be 
considered as work on the system, therefore temperature coupling is essential. Temperature coupling is a critical point in simulations involving deterministic techniques [16]. In the DCV approach the temperature coupling can be applied to the transferring particles in the bulk regions only or to all particles in the simulation box, and also the membrane particles [15] can be exclusively tempered. It is a general experience (although theoretically expectable) that the choice of where and what to thermostate can affect the obtained results. Computation techniques aiming to simulate steady-state diffusion often use the dual control volume grand canonical molecular dynamics (DCV-GCMD) approach [2] to provide constant driving force for diffusion through maintaining concentration gradient between the two control cells (which can also be positioned in a way that a fully periodic simulation box is built from two mirror-image symmetric boxes [17]) by grand canonical, and thus isothermal, Monte Carlo insertion and deletion steps. However, coupling a stochastic method (e.g. a Monte Carlo procedure) to a deterministic one (e.g. MD) is always problematic. For the grand canonical Monte Carlo (GCMC) insertion, it is difficult to choose appropriate starting velocities of the inserted particles and the ratio of the insertion/deletion steps to the MD steps is a crucial parameter (too few MC steps can lead to incorrect results, while too few MD steps can lead to insufficient number of permeation events). On the other hand, for maintaining steady-state flow without standard MC schemes, particles should be created on the feed side and destroyed on the permeate side at the same rate [18]; without using periodic boundary conditions in the direction of the transport, the method requires sufficiently high stationary concentrations of components on the permeate side to uphold the required deletion rate. The NP+LEMC method [9] proposed recently proved to be an efficient technique for transport calculations; it is very fast as compared to other related simulation techniques, and can treat mixtures, but it needs the diffusion coefficient as input parameter. To 
avoid using diffusion coefficients as input here, we introduced a novel technique that couples DMC to LEMC [8]. Because of the stochastic nature of MC, physical time is not explicitly present in these simulations, and only relative fluxes can be determined by this method. Also, the DMC+LEMC method does not produce 'real' MD-like trajectories, only a series of trajectory fragments.

It is important to note that there is a widespread technique called kinetic Monte Carlo method [19], which can deal with longer physical time intervals and (spatially) larger systems in diffusion calculations. Its major and very serious disadvantage is that rate coefficients for all possible moves are required as preliminary information (input parameter).

We can say, in general, that the main problem with simulating steady-state membrane transport at atomic level is that we cannot simulate real size fluid reservoirs to avoid depletion/accumulation of particles in the bulk zones and so particle reinjection/removal is a must in these simulation techniques (for earlier techniques, see e.g. [20, 21]). Nevertheless, the sudden appearance and annihilation of molecules can seriously disturb the steady-state flux of transporting particles. In this work, we developed a simple method to maintain driving force without disturbing significantly the formerly developed steady-state flux. The two main features of our technique are simplicity and connection to macroscopic physical picture of gas transport through membrane. In experimental gas permeations setups pressure is the property that is easily controlled on both the feed and permeate sides of the membrane, so the main governing factor in our method is the pressure.

\section{Methods}

Use of the traditional GCMC approach in a transport simulation implies that non-physical processes/particle moves take place in the immediate vicinity of the membrane. Therefore, it is 
beneficial to put the GCMC control cells far enough away from the membrane. The shift of the control cells away from the membrane region causes the appearance of a new region (so-called access region) between them, where properties such as density and composition of the mixture (or the pressure) cannot be controlled satisfactorily, and this creates unchecked extra resistance to component transport. To avoid these problems, we leave the control cells in contact with the membrane region but the adjustment procedure - responsible for maintaining the bulk-phase properties in the control cell - is restricted to zones far from the membrane region. This means that all properties calculated and monitored in the control cells predominantly stem from the movements and interactions of particles in these regions, and the perturbation is only present in the boundary regions of the simulation cell. Here we consider the total system as being at a constant temperature $T$ with a fixed number of particles $N$ and a box volume $V$. To achieve a target pressure $p$ (or partial pressures in the case of mixtures), regular perturbation in the number of particles is applied close to the boundaries of the simulation box. Since $p$ and $N$ are not conjugate variables, the use of standard statistical-mechanics based molecular simulation operations are not practical. Still, there is a connection between the chemical potential as used in GCMC and the pressure as calculated in our pressure-tuned, boundary-driven molecular dynamics technique (PBD-MD); we found that the pressure can be controlled effectively and unequivocally (although remotely) in the gas phase control cells.

The idea behind our method is based on the fact that in experimental gas permeation arrangements, pressure is the property that can be controlled relatively simply: the partial pressure for each component on the input side of the membrane and the total pressure on the permeate side. The essential point of our scheme is that this pressure control should be realised by adjusting the particle numbers in each control cell indirectly. In contrast to the traditional 
DCV-GCMD approach, instead of doing insertion/deletion in the control regions, these moves are only allowed in the boundary regions, far enough from the membrane region (at the ends of the simulation box). This fact is important, because particle insertions/deletions near the transport region might affect any eventual sorption layers on surfaces of the membrane and can interfere with earlier stabilised steady flux of the permeating particles. The scheme for tuning pressures is kept as simple as possible using essentially a trial and error iterative approach. A particle insertion (disallowing overlap of particles) or deletion step is performed randomly (i.e. randomly chosen particle and position) if the following acceptance criterion is satisfied:

$$
\left|\frac{\left(N_{\text {cortrol coll } \pm 1) \cdot p_{\text {control coll }}}\right.}{N_{\text {control cell }}}-p_{\text {target }}\right|<\left|p_{\text {control cell }}-p_{\text {target }}\right|
$$

where $N_{\text {control cell }}$ is the number of particles in the control cell, and $p_{\text {control cell }}$ and $p_{\text {target }}$ are the calculated and designated pressure in the same control cell, respectively. Here $p$ means partial pressure on the feed side or total pressure on the permeate side and $N_{\text {control cell }}$ denotes the number of particles of the individual components on the feed side or the total number of particles on the permeate side.

\section{Simulation details}

Standard MD was used in the transport simulations employing the leap-frog algorithm as an integrator with the time step of 2 fs. The Berendsen thermostat with the thermal coupling parameter of 200 fs was applied for all particles including particles inside the membrane region; due to the weak coupling, this caused only minor changes in the instantaneous interactions between the particles traveling through and the particles of the membrane lattice. In the steady- 
state system, the temperature was defined by subtracting off the streaming velocity from the $\mathrm{x}$ component of the particle velocities (where $+\mathrm{x}$ direction is the direction of the transport). Random initial velocities, according to the temperature, were assigned to the inserted particles. One particle insertion or deletion attempt was performed periodically in each boundary (wall) region, outside the range of the repulsive walls that close the simulation box in the $\mathrm{x}$ direction. The length of the simulation period of particle number adjustment was taken as 5000 consecutive MD steps and $N_{\text {control cell }}$ and $p_{\text {control cell }}$ were collected as averages for these periods (we experienced that less frequent particle number adjustments cause problems in maintaining the concentrations in the bulk phases, while too frequent adjustments generate exceedingly high perturbations in the system). The average pressure values were calculated by the virial expression.

We present results for the adsorption (equilibrium state) and transport (steady-state) of gas mixtures on silicalite-1 membrane. This is an all-silica zeolite (built up from $\mathrm{SiO}_{4}$ tetrahedrons) with linear and zig-zag channels, which are perpendicular to each other; in both cases the channel diameters are about $0.8 \mathrm{~nm}$. The crystal lattice parameters of its unit cell are $a=$ $2.007 \mathrm{~nm}, b=1.992 \mathrm{~nm}, c=1.342 \mathrm{~nm}$, and the box vectors are mutually orthogonal. The membrane structure was constructed according to available crystallographic information [22] and taken to be defect-free. We built the model structure of silicalite- 1 membrane from 2 unit cells in the $1 \times 1 \times 2$ arrangement for the adsorption calculations and 16 unit cells in the $1 \times 4 \times 4$ arrangement for the transport calculations. These arrangements resulted in a membrane size of $2.007 \mathrm{~nm} \times$ $1.992 \mathrm{~nm} \times 2.684 \mathrm{~nm}$ containing $192 \mathrm{Si}$ and $384 \mathrm{O}$ atoms, and $2.007 \mathrm{~nm} \times 7.968 \mathrm{~nm} \times 5.368 \mathrm{~nm}$ containing $1536 \mathrm{Si}$ and $3072 \mathrm{O}$ atoms, respectively. The particles of the silicalite lattice were 
fixed at the crystallographic positions, so the framework was kept rigid. For membrane diffusion of small-sized gas molecules, this rigid structure is a more or less appropriate assumption [23].

In the case of adsorption simulations, the simulation box size was equal to the size of the constructed membrane (see above) and periodic boundary conditions were applied in all three spatial directions (so the external surface adsorption was not taken into account). In the steadystate transport simulations the box size was set to $80 \mathrm{~nm} \times 7.968 \mathrm{~nm} \times 5.368 \mathrm{~nm}$. It was confined by impenetrable, soft repulsive walls in the direction of the transport, while periodic boundary conditions were applied in the other two directions. The geometry of this simulation box is depicted in Figure 1. The boundary regions were considerably wider than the range of the repulsive wall on both sides of the box. In the case of vacuum conditions on the permeate side the permeate boundary region was set wider.

To describe the molecular interactions, the shifted and cut Lennard-Jones (LJ) pair potential was employed, together with the Lorentz-Berthelot combining rule. The effective interaction potential for a pair of particles $(\alpha$ and $\beta)$ was calculated as

$$
u^{\alpha \beta}(r)=\left\{\begin{array}{ll}
u_{\mathrm{LJ}}^{\alpha \beta}(r)-u_{\mathrm{c}}^{\alpha \beta} & \text { for } r<r_{\mathrm{c}}^{\alpha \beta} \\
0 & \text { for } r<r_{\mathrm{c}}^{\alpha \beta}
\end{array},\right.
$$

where

$$
u_{\mathrm{LJ}}^{\alpha \beta}(r)=4 \varepsilon^{\alpha \beta}\left[\left(\frac{\sigma^{\alpha \beta}}{r}\right)^{12}-\left(\frac{\sigma^{\alpha \beta}}{r}\right)^{6}\right]
$$

is the standard 12-6 LJ potential, $u_{\mathrm{c}}^{\alpha \beta}$ is the value of the potential at the cut-off distance $r_{\mathrm{c}}^{\alpha \beta}$, and $\sigma$ and $\varepsilon$ are the size and energy parameters of the LJ potential, respectively. The spherical cut-off 
distance was set to $3.5 \sigma^{\alpha \beta}$ leading to $u_{\mathrm{c}}^{\alpha \beta}=-0.00217478 \varepsilon^{\alpha \beta}$. All particles interact with the walls according to the Weeks-Chandler-Anderson potential. This is also a shifted and cut LJ potential and has a $2^{1 / 6} \sigma^{\text {wall }}$ cut-off, which is the distance where the LJ potential has its minimum (so the interaction can only be repulsive).

Based on the work of Skoulidas et al. [24], only the interactions of O atoms in the lattice were taken into account. For the multiatomic gas molecules, single-site models were used, and the interaction parameters were taken from the literature. Several size and energy parameters were tested with equilibrium adsorption calculations for $\mathrm{CH}_{4}, \mathrm{CO}_{2}, \mathrm{H}_{2}$ and $\mathrm{N}_{2}$ as singlecomponent gases. We conducted multiple series of GCMC simulation to determine singlecomponent adsorption isotherms and we used these results to select/verify the potential parameters for the pure components (see Table 1).

\section{Results}

In the adsorption calculations the equilibrium selectivity $\left(S_{E}\right)$ was calculated as

$$
S_{\mathrm{E}}=\left(\frac{q_{\mathrm{CO}_{2}}}{q_{i}}\right)_{i \neq \mathrm{CO}_{2}},
$$

where $q$ denotes loading of the silicalite (adsorption inside the adsorbent in $\mathrm{mol} \cdot \mathrm{kg}^{-1}$, relative to the mass of the adsorbent). In the transport calculations, the permeation selectivity (permselectivity or dynamical selectivity) was calculated according to

$$
S_{\mathrm{P}}=\left(\frac{J_{\mathrm{CO}_{2}}}{J_{i}}\right)_{i \neq \mathrm{CO}_{2}},
$$


where $J$ is the component flux $\left(\mathrm{mol} \cdot\left(\mathrm{m}^{2} \cdot \mathrm{s}\right)^{-1}\right)$. For comparison, the idealised permeation ratio $\left(R_{\mathrm{P}}\right)$ was also evaluated; $R_{\mathrm{P}}$ is very similar to $S_{\mathrm{P}}$ but here the pure component fluxes are used and thus it can be regarded as the ideal limit case. (Note that all three properties were calculated to be relative to the values obtained for $\mathrm{CO}_{2}$.) To take the driving force (pressure difference, $\Delta p$ ) between the control cells and the thickness of the membrane $(\Delta x)$ into consideration, we normalised the component flux data $(J)$ with these quantities to obtain the permeance data:

$$
P=J \cdot(\Delta p / \Delta x)^{-1}
$$

The adsorption simulations were conducted in the grand canonical ensemble (fixed chemical potential, volume and temperature) using the equilibrium GCMC method. The pressure/partial pressure of the adsorbate molecules in the gas phase was given indirectly by specifying the component's chemical potential, which was calculated from the ideal gas law.

In the case of the $\mathrm{CH}_{4}$ gas adsorption, the loading increases with the temperature and the pressure in the low pressure region almost linearly, as expected. Relatively good agreement with the experimental data is observed (Figure 2 (a)). Simulations of the $\mathrm{CO}_{2}$ adsorption resulted in similar shapes of the isotherm curves, reproducing the experimentally observed values better at the lower temperatures (Figure 2 (b)). For the $\mathrm{H}_{2}$ gas, experimental data were available at two temperatures. Although the isotherm at $303 \mathrm{~K}$ has an unusual shape, the simulations reproduced the data well. At $338 \mathrm{~K}$, the linear portion of the isotherms (Henry region) is recorded both in simulation and in experiment (Figure 2 (c)). Due to lack of experimental data, we could compare only one isotherm in the case of pure $\mathrm{N}_{2}$. The curves are linear, and an acceptable agreement between simulation and experiment is achieved (Figure 2 (d)). We found simulation data for $\mathrm{N}_{2}$ adsorption on silicalite-1, where the $\mathrm{N}_{2}$ gas was modelled with multiple interaction sites [27]. 
These results were in very good agreement with the data obtained from our simulations and this supported our choice that the one site model for these simple gases is a satisfactory approximation.

We also carried out GCMC simulations to study mixture adsorption: mixtures of $\mathrm{CO}_{2}$ with $\mathrm{CH}_{4}, \mathrm{H}_{2}$ or $\mathrm{N}_{2}$ were used to obtain equilibrium loading and selectivity values for equimolar binary gas systems (more exactly, these are nearly equimolar mixtures, as we set the partial pressures of the components to be equal). In the case of the $\mathrm{CO}_{2}-\mathrm{CH}_{4}$ mixture (Figure 3 (a)) the adsorption of $\mathrm{CH}_{4}$ was partially hindered by the adsorption of $\mathrm{CO}_{2}$ and this yielded $S_{\mathrm{E}}$ values between 3 and 6 (the $S_{\mathrm{E}}$ values are plotted in Figure 6 below). Even more drastic decrease can be observed in the loadings of $\mathrm{H}_{2}$ with the $\mathrm{CO}_{2}-\mathrm{H}_{2}$ gas mixture (Figure 3(b)). The $\mathrm{H}_{2}$ loadings are so low that the calculated $S_{\mathrm{E}}$ values go over 50 (even up to 250). This exclusion of the smaller gas molecules can be attributed to the very different affinities of the $\mathrm{CO}_{2}$ and $\mathrm{H}_{2}$ molecules to the binding sites of the lattice. In the case of the $\mathrm{CO}_{2}-\mathrm{N}_{2}$ mixture (Figure 3(c)), the $S_{\mathrm{E}}$ values (between 10 and 30) follow from an intermediate adsorption rate of $\mathrm{N}_{2}$, as compared to that of $\mathrm{H}_{2}$ or $\mathrm{CH}_{4}$.

In the following, we present results obtained with the PBD-MD technique. The results refer to the cases of single and binary gas transports through silicalite membrane with the $\mathrm{CH}_{4}$, $\mathrm{CO}_{2}, \mathrm{H}_{2}$ and $\mathrm{N}_{2}$ gases, as well as with the binary mixtures of $\mathrm{CO}_{2}$ with the other three components. Before we proceed with our main permeation results, we give a justification of the PBD-MD approach concerning the bulk-phase properties. To ensure that our method uses realistic bulk-phase velocities in the control cells we checked the velocity autocorrelation function for the permeating particles, calculated as follows:

$$
C_{v}(t)=\left\langle v_{i}(0) \cdot v_{i}(t)\right\rangle
$$


This is the average of the scalar products of the starting velocity (from the time of the insertion) and the actual velocity $(v)$ for every transporting particle in the system at time $t$. The particle velocity decorrelates with time ('the particle forgets' its initial velocity), and the decay of $C_{v}$ to nearly zero should happen sufficiently before the time that is needed to reach the membrane. A typical example for $C_{v}$ and the average distance of travel in the direction of the transport $\left(<d_{x}>\right)$ as functions of time are presented in Figure 4. The plots clearly show that the particles practically have no 'memories' of their initial velocities far before they reach the interaction range of the membrane (the minimal travel distance to the membrane is $\sim 30 \mathrm{~nm}$, as seen in Figure 1). For comparison, the average fluxes in this system were about 0.180 and $0.173 \mathrm{~ns}^{-1} \mathrm{~nm}^{-2}$ for $\mathrm{CO}_{2}$ and $\mathrm{CH}_{4}$ respectively.

The concentration profile of the transporting particles is also a good indicator whether the control cells can be regarded as bulk phases or not. In our simulations there are constant concentrations in the dominant part of the control cell for each component. Illustrating this, representative concentration profiles are shown in Figure 5 for a couple of systems. In the case of mixtures (5 (b), 5 (c)) the partial pressures on the feed side are identical and thus the concentrations become also, more or less, equal to each other. If vacuum condition is set on the permeate side, the obtained pressure is not properly zero (correct vacuum cannot be achieved). This is caused by the nature of the technique, namely that the particles are deleted in a region far from the membrane. This behaviour is also present in the common gas permeation measurements at microscopic scale, so we took no further efforts to change this character of the technique. It is also notable that for $p=100 \mathrm{kPa}$ permeate pressure (e.g. 5 (c)), the obtained total pressure was always correct in the simulations, even if the partial pressures (and component concentrations) were different, corresponding to the selectivity related properties of the membrane. With the 
present pressure tuning procedure and length of the period of particle number adjustment, we could reproduce the target pressures within 1-3\% in both control cells of the investigated systems (certainly, the appropriate length of the period of adjustment can be different in other systems).

We compare our transport results for pure components with available experimental data obtained by the pressure drop method [30] at atmospheric pressure (1 bar) on the permeate side in Table 2. The calculated and the measured permeance data $P$ and the idealised permeation ratio values $R_{\mathrm{P}}$ agree relatively well; however, the simulated $R_{\mathrm{P}}$ values show cumulative deviations from the experimental ones based on the deviations of the independent permeance results. The applied shifted and cut LJ potential is a less detailed interaction model and thus only a guess for the real system. As seen above, even the experimental isotherms of the pure gases cannot be exactly reproduced using the selected literature potential parameters. Moreover, the experimentally measured data can be disturbed by the quality of the adsorbent/membrane (contamination or defects in the crystal structure, and presence of supporting layer). In light of these, we consider the calculated permeance data to be in a relatively good agreement with the experimentally observed ones.

$R_{\mathrm{P}}$ from single component transport simulations provides estimation for the separation efficiency of the membrane in mixture transport assuming that the fluxes of the components are totally separated and not affected by each other. In some cases, this assumption holds and $R_{\mathrm{P}}$ can be a good approximation, however, in a number of cases permeation rate values calculated from simulations of mixtures are significantly different, meaning that the transport of the mixture is realised by momentum coupling of the components passing through the membrane. The difference between $R_{\mathrm{P}}$ and $S_{\mathrm{P}}$ is clearly shown by the results from simulations with the $\mathrm{CO}_{2}-\mathrm{CH}_{4}$ mixture at vacuum conditions on the permeate side (Figure 6 (a)): in contrast to the $S_{\mathrm{P}}$ values, the 
$R_{\mathrm{P}}$ values exhibit milder temperature dependence. This behaviour is attributed to the fact that in the mixture transport, the flux of $\mathrm{CH}_{4}$ increases with the temperature due to its augmented relative adsorption ratio (at the expense of the adsorption of $\mathrm{CO}_{2}$ ). This latter is indicated by the trends in the $S_{\mathrm{E}}$ data here, although these data, which do not include contributions from the dynamics of the particle movements, are significantly higher than their dynamic counterparts. We must note, however, that the feed side pressure in the dynamic simulations cannot be unequivocally compared to the equilibrium pressure of the adsorption simulations. The equilibrium adsorption loadings proved to be consistently larger than the loadings of the membrane in dynamic simulations with $p_{\text {feed }}=p_{\text {adsorption }}$.

While almost no temperature and pressure dependences of $R_{\mathrm{P}}$ can be seen in Figure 6 (b), rapid changes in $S_{\mathrm{P}}$, from $\sim 2.5$ to $\sim 0.5$ within a $100 \mathrm{~K}$ range, are obtained for the equimolar $\mathrm{CO}_{2^{-}}$ $\mathrm{H}_{2}$ mixture. This behaviour is caused by the blocking of the faster moving $\mathrm{H}_{2}$ molecules by the tightly adsorbed $\mathrm{CO}_{2}$ molecules with smaller kinetic energy. Adsorbing to silicalite, $\mathrm{CO}_{2}$ has higher heat of adsorption than $\mathrm{H}_{2}$, resulting in higher temperature dependence of the loadings of $\mathrm{CO}_{2}$ (see also Figure 2 (b), (c)). It is remarkable here that every equilibrium selectivity value is by two orders of magnitude higher than the corresponding dynamic one. Similar behaviour is obtained for $S_{\mathrm{P}}$ from the simulations with the $\mathrm{CO}_{2}-\mathrm{N}_{2}$ mixture (Figure 6 (c)), while the data of $R_{\mathrm{P}}$ show slightly larger temperature dependence as compared to those obtained with the $\mathrm{CO}_{2}-\mathrm{H}_{2}$ mixture. Again, the $S_{\mathrm{E}}$ curves underline the preferred adsorption of $\mathrm{CO}_{2}$ over $\mathrm{N}_{2}$. Setting the permeate side pressure to $100 \mathrm{kPa}$, we have an opportunity to compare our results with reliable experimental data for the $\mathrm{CO}_{2}-\mathrm{H}_{2}$ system [31]. Figure 6 (d) illustrates the matching between simulation and measurement and, at the same time, reveals a slight change in the curves with respect to the vacuum case (Figure $6(b)$ ). 
As our last tests, we performed simulations with pure $\mathrm{H}_{2}$ and $\mathrm{CO}_{2}$ at $338 \mathrm{~K}$ and $p_{\text {feed }}=200$ $\mathrm{kPa}$ by doubling the membrane thickness $(4.014 \mathrm{~nm}$ in the transport direction, so the pressure gradient is about $50 \mathrm{kPa} / \mathrm{nm}$ in the vacuum case). The obtained flux data for $\mathrm{H}_{2}$ were roughly half of the ones calculated for the original membrane thickness in both cases of the permeate-side pressure (vacuum or $100 \mathrm{kPa}$ ). For $\mathrm{CO}_{2}$, however, the flux data reduced only to $65-70 \%$ of the corresponding reference data. This seemingly anomalous behaviour is apparently due to the comparatively high loading of the membrane with $\mathrm{CO}_{2}$ molecules and the developed surface layers on the membrane. The investigation of this problem will be the topic of our future studies.

\section{Conclusions}

In this work, we propose a novel MD-based simulation technique for membrane transport studies. The method allows accurate determination of stationary fluxes of gases through mesoporous and microporous membranes as it properly mimics the common experimental situation in gas permeations measurements, where the main control parameter is the pressure (pressure drop methods). The key feature of the method is that the necessary perturbation to maintain steady state is applied only at the boundary regions of the simulation cell. We verified the method by checking that the applied perturbation is sufficiently far away from the membrane and the bulkphase properties are well controlled in a simulation cell of common size.

We established that the pressure adjustment procedure described here works correctly for gaseous bulk phases (certainly, the more dilute a gas in the simulation is, the more simple the pressure adjustment is). One of the most advantageous features of the method is that both the total pressure and the partial pressures can be controlled efficiently. Fixing the total pressure on the permeate side, as it can be the case in normal gas permeations measurements, is not a trivial task in other approaches, where, directly or indirectly, the chemical potentials of the individual 
components are set to predefined values. As the permeate side might have a considerable feedback effect to the microscopic transport, the total pressure adjustment is a key operation (a small permeate reservoir can influence the transport not only through the thermodynamic driving force but, e.g. via kinetic blocking of the surface pores once larger particles can diffuse from this side into the membrane). Using the individual chemical potentials instead of the total pressure as control parameters on the output side of the membrane, the system (i.e. the regulation of the transfer process) becomes overdetermined, except for the case of vacuum conditions. Nevertheless, our scheme allows using the chemical potentials as control parameters on the input side (instead of the partial pressures), since tuning the chemical potentials can be realised similarly by measuring their values in the control cells (e.g. via the Widom test particle method). Certainly, the scheme can have some drawbacks as well. Larger statistical errors of the pressure measurements in these simulations can lead to incorrect pressure adjustment, and therefore, it is important to properly handle the long range corrections, especially for systems containing charged particles. Possible obstacles include the inefficient particle insertions in denser phases and/or with multisite (rigid or flexible) gaseous molecules, and the treatment of electroneutrality in particle insertions-deletions with charged species.

Our investigations imply that atomistic simulations with commonly used system sizes have difficulties in accurately reproducing results of transport experiments despite the fact that much progress has been made in developing techniques in the last decade, primarily in modelling the fluid reservoirs. Until we cannot simulate sufficiently large systems with membrane thickness close enough to the real size, only order of magnitudes of transport properties and their trends can be predicted reasonably. In this sense, we consider our present transport simulation results (trends and orders of magnitudes) to be in satisfactory agreement with available data of transport 
experiments. Undoubtedly, by using more realistic potential models and tuning their potential parameters, a better agreement could be achieved with the transport data. Our preliminary simulations with the flexible counterpart of the model framework, e.g. showed that the introduction of a realistic flexibility to the silicalite membrane gives rise to a slight increase in the fluxes but does not cause a considerable change in the selectivities (at the same time, however, it largely increases the computational demand of the simulations). Consequently, for the investigated mixtures, the most important trend that is revealed from the present molecular simulations is the consistently lower values of the transport selectivity with respect to the equilibrium ones: the prevalent adsorption of $\mathrm{CO}_{2}$ observed in all cases not always resulted in transport selectivity in favour of $\mathrm{CO}_{2}$.

\section{Acknowledgments}

We gratefully acknowledge the support of the Hungarian National Research Fund (OTKA NN113527) in the framework of ERA Chemistry and the financial and infrastructural support of the Hungarian State and the European Union under TAMOP-4.2.2.A-11/1/KONV-2012-0071.

\section{References}

[1] Freeman BD. Basis of Permeability/Selectivity Tradeoff Relations in Polymeric Gas Separation Membranes. Macromolecules 1999;32:375-380.

[2] Heffelfinger GS, van Swol F. Diffusion in Lennard-Jones fluids using dual control volume grand canonical molecular dynamics simulation (DCV-GCMD). J Chem Phys. 1994;100:7548.

[3] Im W, Seefeld S, Roux B. A Grand Canonical Monte Carlo-Brownian dynamics algorithm for simulating ion channels. Biophys J. 2000;79:788-801.

[4] Lísal M, Brennan JK, Smith WR, Siperstein FR. Dual control cell reaction ensemble molecular dynamics: A method for simulations of reactions and adsorption in porous materials. J Chem Phys. 2004;121: 4901-4912. 
[5] Rutkai G, Kristóf T. Dynamic Monte Carlo simulation in mixtures. J Chem Phys. 2010;132: 124101.

[6] Rutkai G, Boda D, Kristóf T. Relating binding affinity to dynamical selectivity from dynamic Monte Carlo simulations of a model calcium channel. J Phys Chem Lett. 2010;1: 21792184.

[7] Csányi É, Boda D, Gillespie D, Kristóf T. Current and selectivity in a model sodium channel under physiological conditions: Dynamic Monte Carlo simulations. Biochim Biophys Acta. 2012;1818:592-600.

[8] Ható Z, Boda D, Kristóf T, Simulation of steady-state diffusion: Driving force ensured by dual control volumes or local equilibrium Monte Carlo. J Chem Phys. 2012;137:054109.

[9] Boda D, Gillespie D. Steady-state electrodiffusion from the Nernst-Planck equation coupled to local equilibrium Monte Carlo simulations. J Chem Theor Comput. 2012;8:824-829.

[10] Maginn EJ, Bell AT, Theodorou DN. Transport diffusivity of methane in silicalite from equilibrium and nonequilibrium simulations. J Phys Chem. 1993;97:4173-4181.

[11] Salih A. Molecular simulation of the adsorption and transport properties of carbon dioxide, methane, water and their mixtures in coal-like structures [dissertation]. London, UK: Imperial College London; 2010.

[12] Huang C, Nandakumar K, Choi PYK, Kostiuk LW. Molecular dynamics simulation of a pressure-driven liquid transport process in a cylindrical nanopore using two self-adjusting plates. J Chem Phys. 2006;124:234701

[13] Evans DJ, Morriss OP. Non-Newtonian molecular dynamics. Comput Phys Rep1984;1:297343. 297-343.

[14] Zhu F, Tajkhorshid E, Schulten K. Pressure-induced water transport in membrane channels studied by molecular dynamics. Biophys J. 2002;83:154-160.

[15] Frentrup H, Avendaño C, Horsch M, Salih A, Müller EA. Transport diffusivities of fluids in nanopores by non-equilibrium molecular dynamics simulation. Mol Sim. 2012;38:540553.

[16] Yong X, Zhang LT. Thermostats and thermostat strategies for molecular dynamics simulations of nanofluidics. J Chem Phys 2013;138:084503 
[17] Furukawa S. Shigeta T. Nitta T. Non-equilibrium molecular dynamics for simulating permeation of gas mixtures through nanoporous carbon membranes. J Chem Eng Japan 1996;29: 725-728

[18] Delgado-Buscalioni R. Coveney PV. USHER: An algorithm for particle insertion in dense fluids. J Chem Phys 2003;119: 978-987

[19] Auerbach SM, Jousse F, Vercauteren DP. Dynamics of sorbed molecules in zeolites. In: Catlow CRA, van Santen RA, Smit B, editors. Computer modelling of microporous and mesoporous materials. London: Academic Press; 2004. p. 49-108.

[20] Kjelstrup S, Bedeaux D, Inzoli I, Simon JM. Criteria for validity of thermodynamic equations from non-equilibrium molecular dynamics simulations. Energy. 2008;33:11851196.

[21] Xu J, Kjelstrup S, Bedeaux, D. Molecular dynamics simulations of a chemical reaction; conditions for local equilibrium in a temperature gradient. Phys Chem Chem Phys. 2006;8:2017-2027.

[22] Olson DH, Kokotailo GT, Lawton SL, Meier WM. Crystal structure and structure-related properties of ZSM-5. J Phys Chem. 1981;85:2238-2243.

[23] Demontis P, Fois ES, Suffritti GB, Quartieri S. Molecular dynamics studies on zeolites. 4. Diffusion of methane in silicalite. J Phys Chem. 1990;94:4329-4334.

[24] Skoulidas AI, Sholl DS. Transport Diffusivities of $\mathrm{CH}_{4}, \mathrm{CF}_{4}, \mathrm{He}, \mathrm{Ne}, \mathrm{Ar}, \mathrm{Xe}$, and $\mathrm{SF}_{6}$ in silicalite from stomistic simulations. J Phys Chem B. 2002;106:5058-5067.

[25] Takaba H, Matsuda E, Nakao S. Correlation of temperature dependence of gas permeability with pore size in molecular sieving membranes: a grand canonical ensemble molecular dynamics study. J Phys Chem B. 2004;108:14142-14147.

[26] Liu J, Culp JT, Natesakhawat S, Bockrath BC, Zande B, Sankar SG, Garberoglio G, Johnson JK. Experimental and theoretical studies of gas adsorption in $\mathrm{Cu}_{3}(\mathrm{BTC})_{2}$ : an effective activation procedure. J Phys Chem C. 2007;111:9305-9313.

[27] Makrodimitris K, Papadopoulos GK, Theodorou DN. Prediction of permeation properties of $\mathrm{CO}_{2}$ and $\mathrm{N}_{2}$ through silicalite via molecular simulations. J Phys Chem B. 2001;105:777788.

[28] Wiraman SK, Petersson M, Creaser D. Adsorption of $\mathrm{CO}, \mathrm{CO}_{2}$ and $\mathrm{H}_{2}$ in silicalite-1: measurements and simulations. AJChE. 2008;8:9-19. 
[29] Zhu W, Hrabanek P, Gora L, Kapteijn F, Moulijn JA. Role of adsorption in the permeation of $\mathrm{CH}_{4}$ and $\mathrm{CO}_{2}$ through a silicalite-1 membrane. Ind Eng Chem Res. 2006;45:767-776.

[30] Matsufuji T, Nishiyama N. Ueyama K, Matsukata M. Permeation characteristics of butane isomers through MFI-type zeolitic membranes. Catal Today. 2000;56:265-273.

[31] Wirawan SK, Creaser D, Lindmark J, Hedlund J, Bendiyasa IM, Sediawan WB. $\mathrm{H}_{2} / \mathrm{CO}_{2}$ permeation through a silicalite-1 composite membrane. J Membrane Sci. 2011;375:313322. 
Table 1. Effective pair potential parameters (size and energy) used in the simulations.

\begin{tabular}{ccc}
\hline \hline & $\sigma / \mathrm{nm}$ & $\left(\varepsilon / k_{\mathrm{B}}\right) / \mathrm{K}$ \\
\hline O atoms in silicalite [24] & 0.27 & 120 \\
$\mathrm{CH}_{4}[24]$ & 0.373 & 147.9 \\
$\mathrm{CO}_{2}[25]$ & 0.3941 & 195.2 \\
$\mathrm{H}_{2}[26]$ & 0.296 & 34.2 \\
$\mathrm{~N}_{2}[26]$ & 0.3572 & 93.98 \\
Soft repulsive wall & 0.3 & 120 \\
\hline \hline
\end{tabular}


Table 2. Permeance $(P)$ and idealised permeation ratio $\left(R_{\mathrm{P}}\right)$ values obtained from simulations and experiments [31] with the pure $\mathrm{H}_{2}$ and $\mathrm{CO}_{2}$ gases at $100 \mathrm{kPa}$ on the permeate side.

\begin{tabular}{|c|c|c|c|c|c|c|}
\hline & $\begin{array}{c}\mathrm{H}_{2} \\
\text { Simulation }\end{array}$ & $\begin{array}{c}\mathrm{H}_{2} \\
\text { Experiment }\end{array}$ & $\begin{array}{c}\mathrm{CO}_{2} \\
\text { Simulation }\end{array}$ & $\begin{array}{c}\mathrm{CO}_{2} \\
\text { Experiment }\end{array}$ & Simulation & Experiment \\
\hline $\mathrm{p} / \mathrm{kPa}$ & \multicolumn{4}{|c|}{$303 \mathrm{~K}, \mathrm{P} /\left(\mathrm{nm} \cdot \mathrm{mol} \cdot \mathrm{m}^{-2} \cdot \mathrm{s}^{-1} \cdot \mathrm{bar}^{-1}\right)$} & \multicolumn{2}{|c|}{$303 \mathrm{~K}, R_{\mathrm{p}}$} \\
\hline 140 & 1676 & 1107 & 542 & 716 & 0.323 & 0.646 \\
\hline 200 & 1487 & $1148^{*}$ & 669 & $662^{*}$ & 0.450 & 0.576 \\
\hline 250 & 1472 & - & 545 & - & 0.371 & - \\
\hline $\mathrm{p} / \mathrm{kPa}$ & \multicolumn{4}{|c|}{$338 \mathrm{~K}, \quad P /\left(\mathrm{nm} \cdot \mathrm{mol} \cdot \mathrm{m}^{-2} \cdot \mathrm{s}^{-1} \cdot \mathrm{bar}^{-1}\right)$} & \multicolumn{2}{|c|}{$338 \mathrm{~K}, R_{\mathrm{p}}$} \\
\hline 140 & 1338 & 1013 & 540 & 540 & 0.403 & 0.533 \\
\hline 200 & 1362 & $1026^{*}$ & 535 & $574^{*}$ & 0.392 & 0.559 \\
\hline 250 & 1322 & - & 473 & - & 0.357 & - \\
\hline $\mathrm{p} / \mathrm{kPa}$ & \multicolumn{4}{|c|}{$408 \mathrm{~K}, \quad \mathrm{P} /\left(\mathrm{nm} \cdot \mathrm{mol} \cdot \mathrm{m}^{-2} \cdot \mathrm{s}^{-1} \cdot \mathrm{bar}^{-1}\right)$} & \multicolumn{2}{|c|}{$408 \mathrm{~K}, R_{\mathrm{p}}$} \\
\hline 140 & 903 & 743 & 429 & 405 & 0.475 & 0.545 \\
\hline 200 & 1025 & $790^{*}$ & 328 & $425^{*}$ & 0.320 & 0.538 \\
\hline 250 & 1082 & - & 393 & - & 0.363 & - \\
\hline
\end{tabular}

The applied membrane pressure difference was $80 \mathrm{kPa}$ instead of $100 \mathrm{kPa}$ 


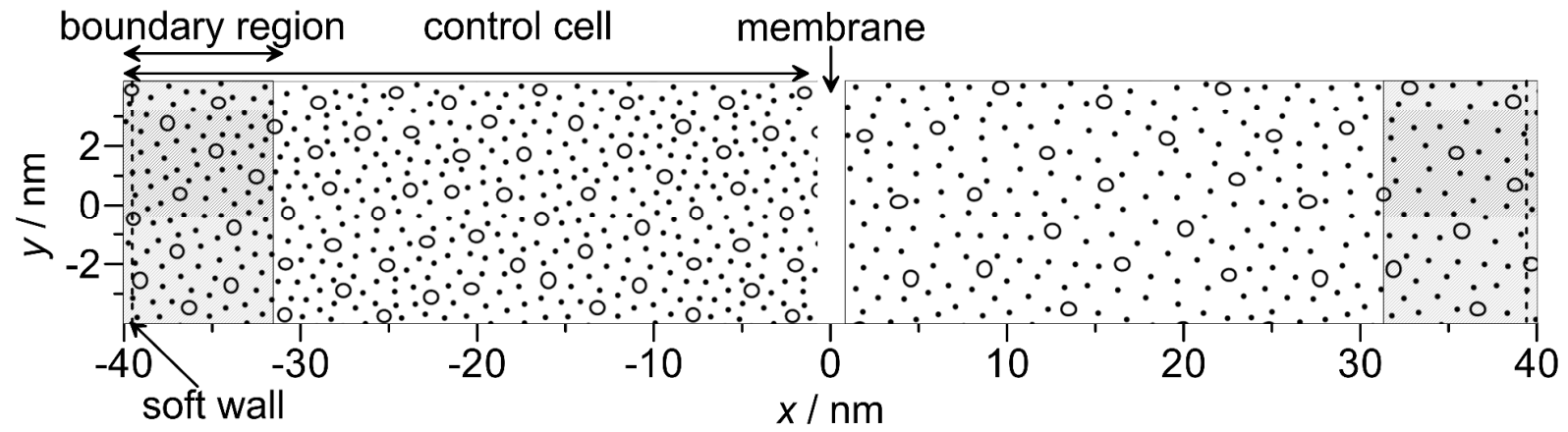

Figure 1. Arrangement of a typical PBD-MD simulation. 


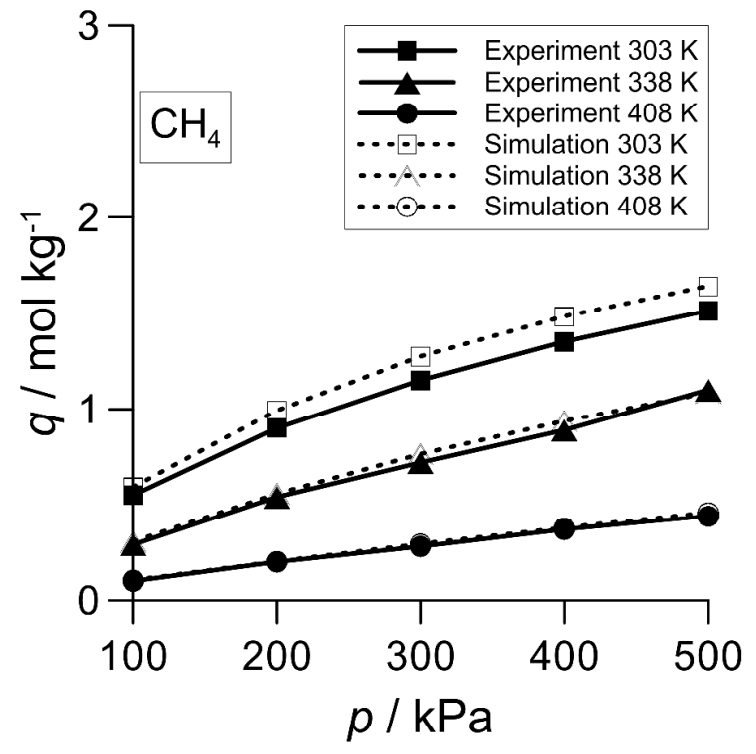

(a)

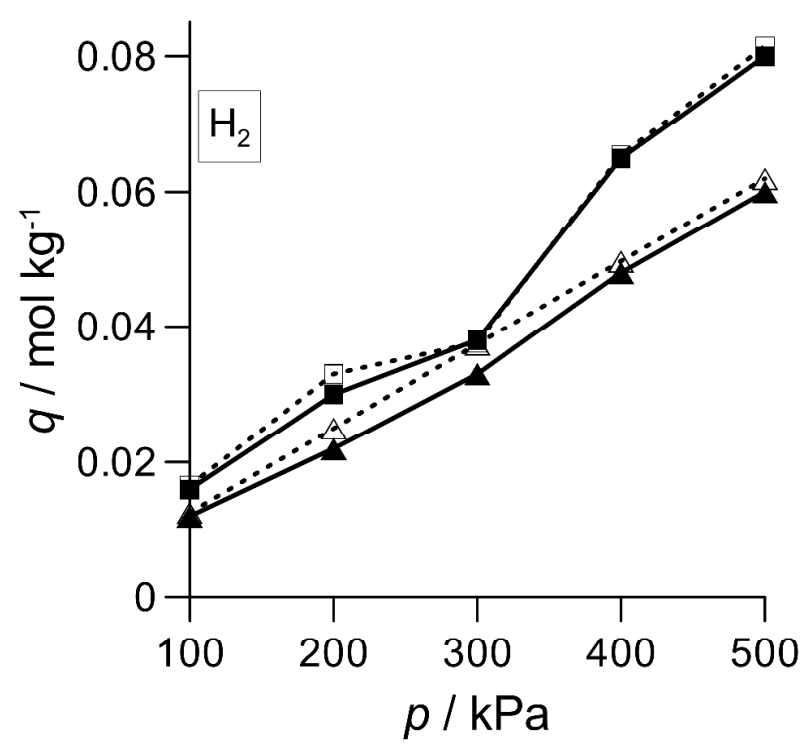

(c)

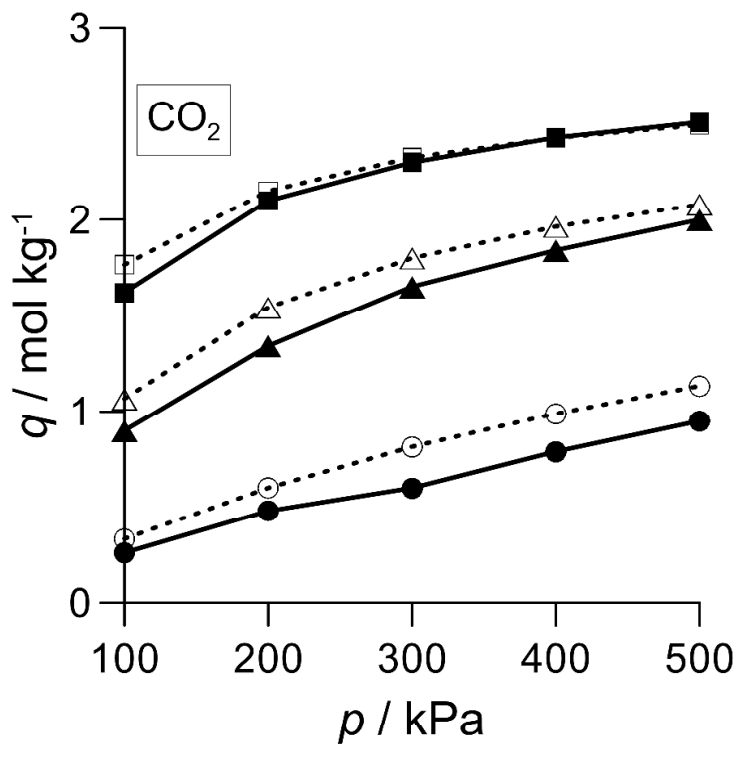

(b)

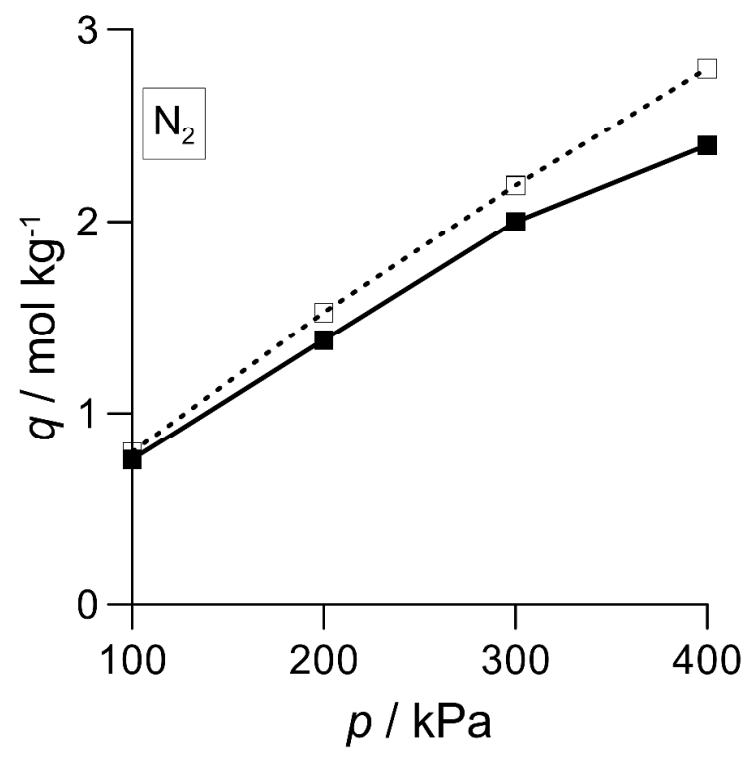

(d)

Figure 2. Adsorption isotherms of $\mathrm{CH}_{4}, \mathrm{CO}_{2}, \mathrm{H}_{2}$ and $\mathrm{N}_{2}$ on silicalite-1. The experimental data are taken from Refs. [27-29] and Ref. [31]. 


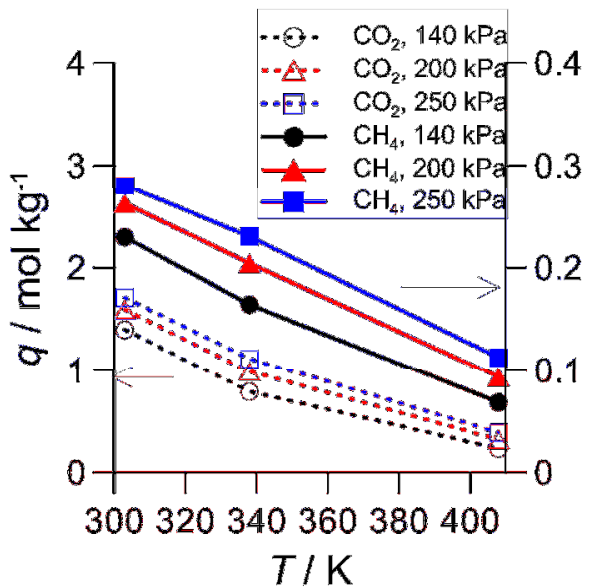

(a)

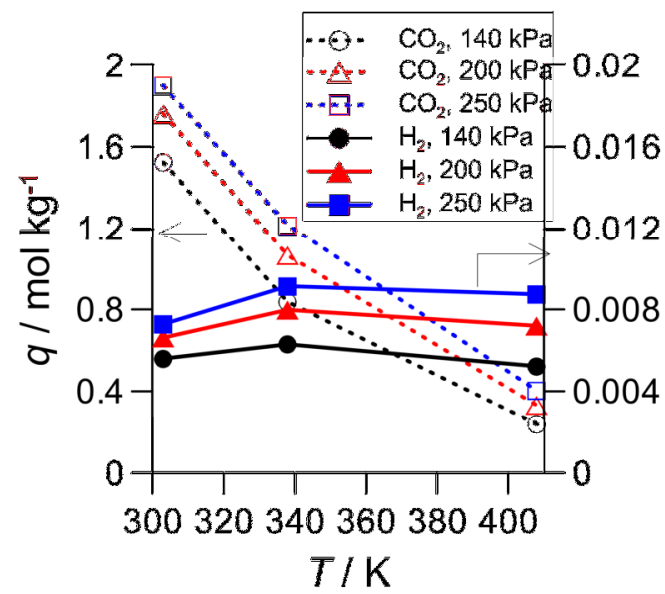

(b)

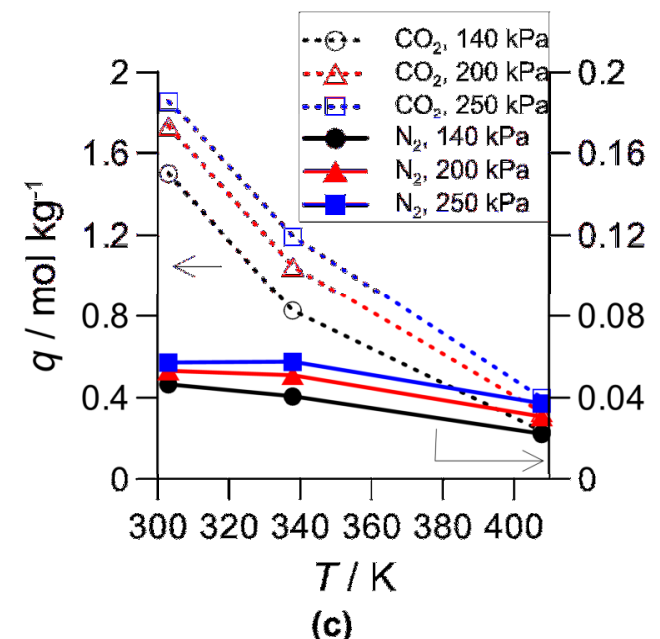

Figure 3. Loadings of silicalite obtained from simulations using equimolar $\mathrm{CO}_{2}-\mathrm{CH}_{4}, \mathrm{CO}_{2}-\mathrm{H}_{2}$ and $\mathrm{CO}_{2}-\mathrm{N}_{2}$ mixtures. 

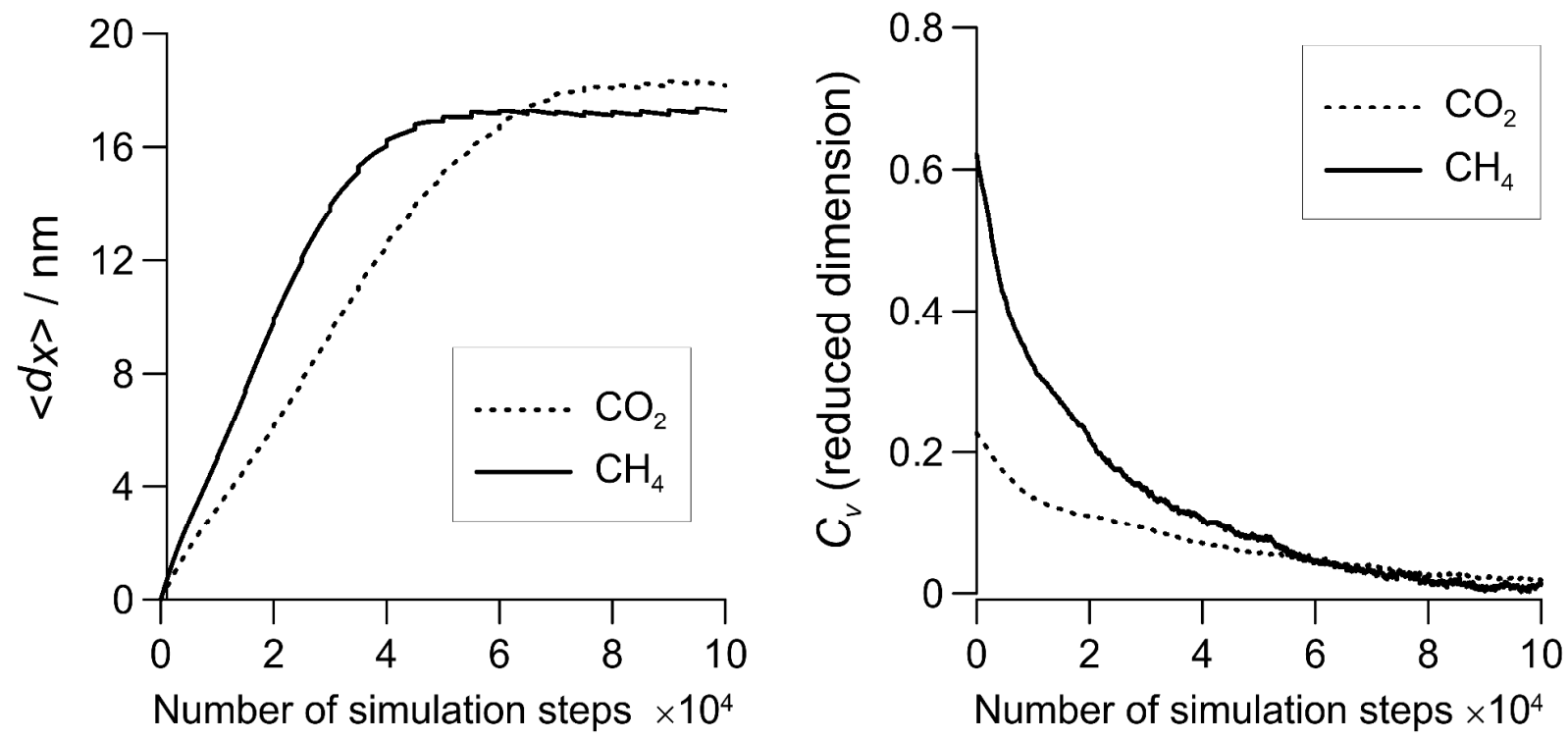

Figure 4. Average distance of travel in the direction of the transport and velocity autocorrelation function of the transporting components for $\mathrm{CO}_{2}-\mathrm{CH}_{4}$ systems at $338 \mathrm{~K}$ with $p=200 \mathrm{kPa}(100$ $\mathrm{kPa}$ for both components) on the feed side and vacuum on the permeate side. 


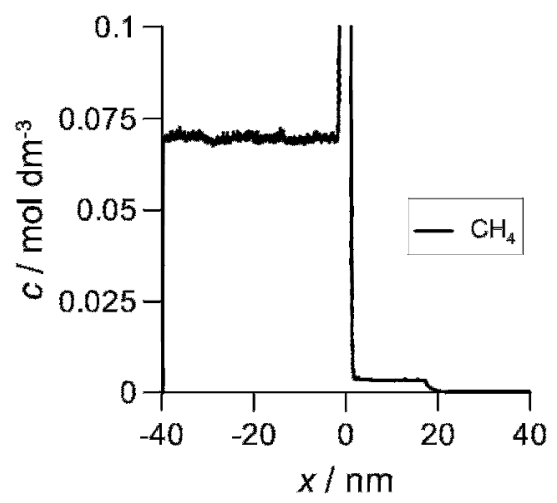

(a)

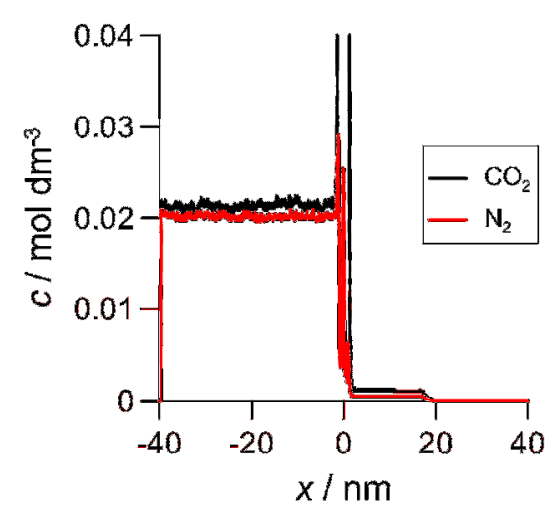

(b)

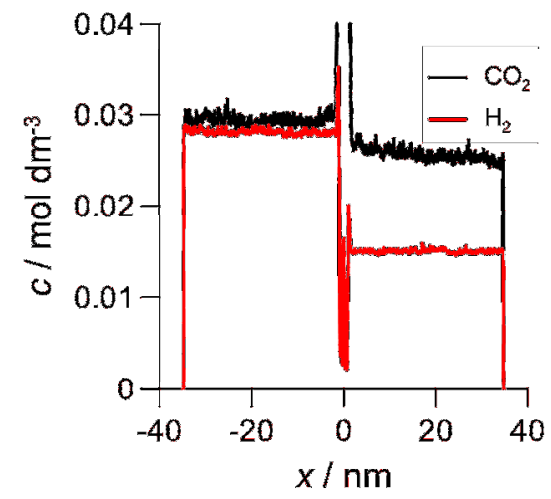

(c)

Figure 5. Steady-state concentration profiles in the transport simulations: (a) pure $\mathrm{CH}_{4}$ at $338 \mathrm{~K}$ with $p=200 \mathrm{kPa}$ on the feed side and vacuum on the permeate side, (b) $\mathrm{CO}_{2}-\mathrm{N}_{2}$ mixture at $408 \mathrm{~K}$ with $p=140 \mathrm{kPa}$ (70 $\mathrm{kPa}$ for both components) on the feed side and vacuum on the permeate side, (c) $\mathrm{CO}_{2}-\mathrm{H}_{2}$ mixture at $303 \mathrm{~K}$ with $p=140 \mathrm{kPa}$ (70 kPa for both components) on the feed side and $p=100 \mathrm{kPa}$ on the permeate side. For example, $0.1 \mathrm{~mol} \mathrm{dm}^{-3}$ means $\sim 100$ particles in our typical feed-side reservoir with a length of $\sim 40 \mathrm{~nm}$. 


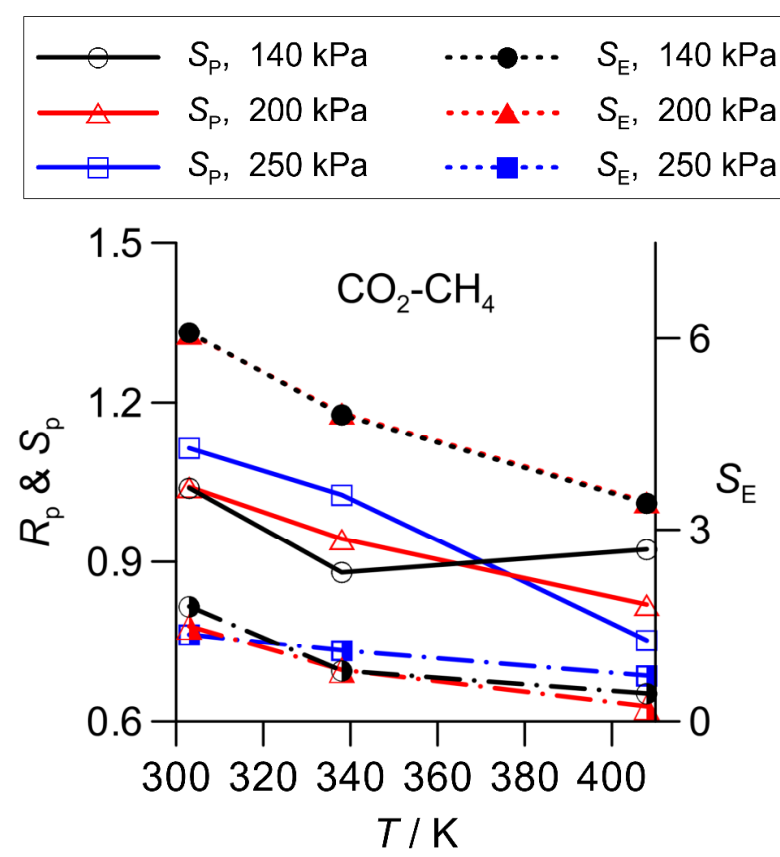

(a)

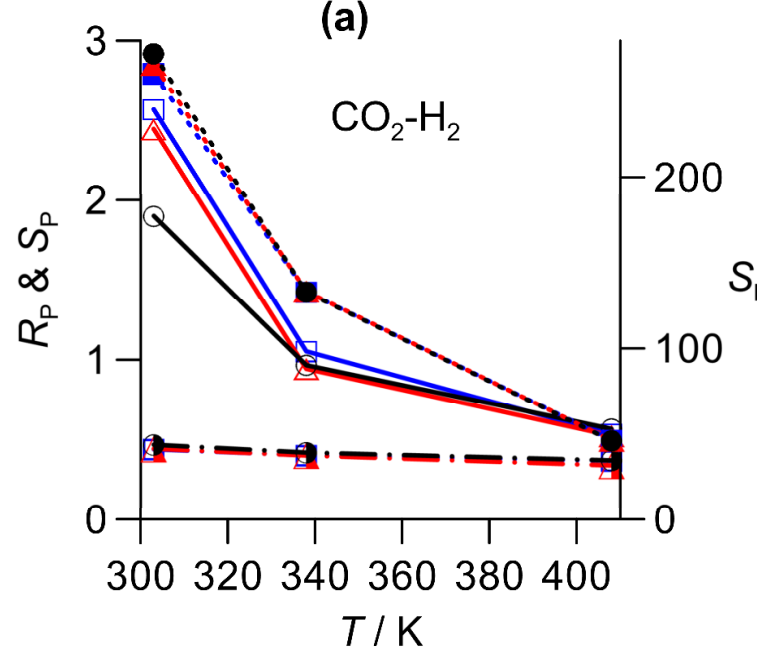

(b)

$\begin{array}{llll}\text { - } & \cdot R_{\mathrm{P}}, 140 \mathrm{kPa} \\ \text { —- } & R_{\mathrm{P}}, 200 \mathrm{kPa} \\ \text { —- } & R_{\mathrm{P}}, 250 \mathrm{kPa}\end{array}$

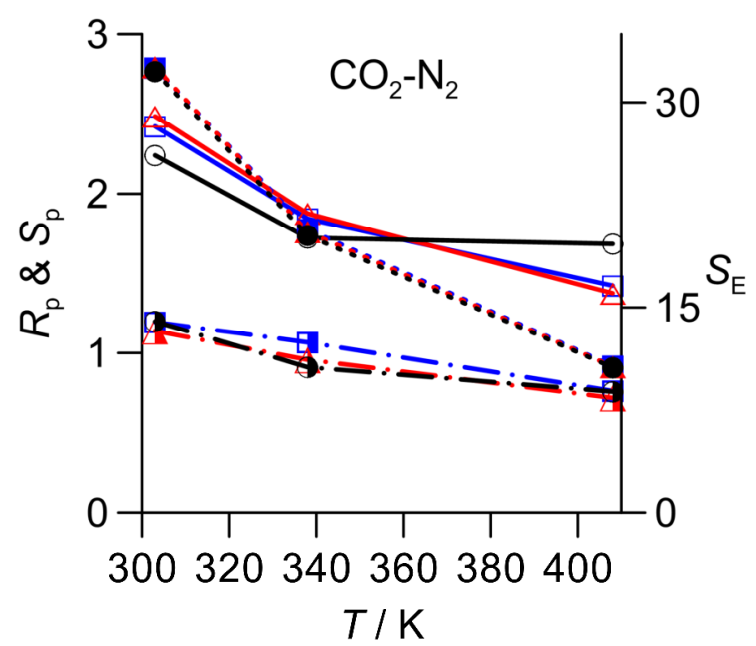

(c)

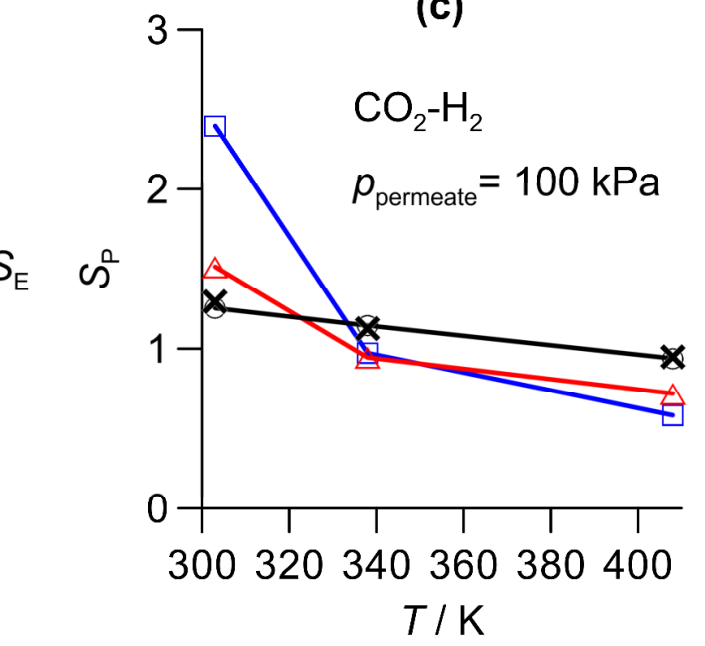

(d)

Figure 6. Idealised permeation ratios $\left(R_{\mathrm{P}}\right)$ compared to permeation selectivities $\left(S_{\mathrm{P}}\right)$ in the case of equimolar $\mathrm{CO}_{2}-\mathrm{CH}_{4}, \mathrm{CO}_{2}-\mathrm{H}_{2}$ and $\mathrm{CO}_{2}-\mathrm{N}_{2}$ mixtures and the equilibrium selectivity $\left(S_{\mathrm{E}}\right)$ data from adsorption calculations. For (a)-(c), there is a vacuum condition and, for (d), $p=100 \mathrm{kPa}$ on the permeate side (experimental data from [31] are also plotted here). 